\title{
A Comprehensive Study on the Impact of Various Nano-gratings on MSM-PDs for Enhancement in the Light Absorption
}

\author{
Alif Islam, Narottam Das, Mohammad Mohiuddin Uzzal
}

\begin{abstract}
In this paper, we have analyzed metalsemiconductor-metal photodetectors (MSM-PDs) with different nano-grating structures or shapes to improve the light absorption capacity into the device in details for high-speed communication systems and networks. The plasmonic-based MSM-PD structure demonstrates a significant improvement in light absorption capacity for the developed device compared to conventional MSMPDs i.e., devices that have not employed the nano-gratings. The light absorption capacity of the device is varied with the variation of geometrical shapes and parameters of the nano-gratings, such as the nano-grating height, slit width and so on. These nanograting structures are assisting in light transmission through the central slit (i.e., subwavelength apertures) efficiently, resulting in the excitation of surface plasmon polaritons (SPPs) as the incident photons interact with the nano-gratings/ nano-corrugations. This improved light transmission in the central slit along with excited SPPs results in resonant light absorption in the device. This means the light trapped inside the central slit is triggered by the SPPs to a higher order magnitude. This causes the light absorption enhancement for the device, i.e., more light is transmitted through the device instead of reflecting back to the surface. The simulation results demonstrated that the light absorption enhancement factor (LAEF) for these devices have improved dramatically due to the nano-gratings. For modeling and simulation of these devices, OptiFDTD tool is used which is based on finite difference time domain (FDTD) method. The application of these simulated devices is in the range of 800-850- $\mathrm{nm}$. The simulation results are suitable for the design of nano-structured MSM-PDs that can be used in highspeed communication systems and sensor network systems.
\end{abstract}

Keywords - Finite difference time domain (FDTD) method, Light absorption enhancement factor (LAEF), Plasmonic-based MSM-PDs, Subwavelength aperture, Surface plasmon polaritons (SPPS).

\section{Alif Islam}

Graduate Student; Dept. of EEE

American International University-Bangladesh (AIUB)

Dhaka, Bangladesh

Email: alif.islam.bma@gmail.com

\section{Narottam Das}

Senior Lecturer; School of Engineering and Technology

Central Queensland University

Melbourne, Australia

Email: n.das@cqu.edu.au

\author{
Mohammad Mohiuddin Uzzal \\ Professor; Dept. of EEE \\ American International University-Bangladesh (AIUB) \\ Dhaka, Bangladesh \\ Email: drmohiuddin@aiub.edu
}

\section{INTRODUCTION}

In recent years, the development in optical and electrical components led to new doorways for ultra-high speed communication systems and networks. There have been several optimization and miniaturization procedures taken place for optical and electronic devices following the trend of the semiconductor industry, i.e., Moore's law, over the last three decades. These rapid growths, in device technology, resulted in highly efficient devices that can contribute to high-speed communication systems, ultra-fast chip-to-chip interconnects, and faster network systems. In this regard, photodetectors are one of the most important parts of any ultra-fast communication systems or networks. Over the decades, numerous researches have been taken place regarding the development of photodetectors, by analyzing their photonic behavior under different circumstances. Metal-semiconductor-metal photodetectors (MSM-PDs) are an ideal candidate for ultra-high-speed communication systems or networks because of their efficient light capturing performance, simpler construction, and easy fabrication compared to other photodetectors, such as phototransistors, avalanche photo-detectors or diodes, and positive-intrinsic-negative (PIN) diodes etc. [1-10].

Fig. 1 represents a simplified diagram of a conventional MSM-PD, i.e., without the nano-gratings on top of the subwavelength apertures. The MSM-PDs mainly employ two Schottky contacts, i.e., two identical metallic contacts (i.e. electrodes) on top of a semiconductor substrate, resulting in two separate junctions, one with forward-bias and other with reversebias [11-16]. During regular operation of this device, it was observed that a large amount of incident light was reflected from the device surface and hence resulting in lower light transmission to the active region of the device [3]. With the introduction of plasmonic nanostructures on top of the device surface, it is possible to reduce the light reflection issue considerably, because of the nano-gratings able to work as a lens to collect or welcome the light efficiently and transmit it to the central slit of the device [2]. These plasmonic nano-gratings are placed on top of the two metallic contacts (i.e., subwavelength apertures) in a periodic manner. This results in the enhancement of light absorption in the device and improves the responsivity, spectral response, and quantum efficiency of the MSM-PDs [17$19]$.

Fig. 2 shows the diagram of a rectangular-shaped nanostructured MSM-PD with a grating height of $100-\mathrm{nm}$, slit width of $50-\mathrm{nm}$ and grating period of $810-\mathrm{nm}$. This type of photodetector has a higher light absorption enhancement factor 
(LAEF) compared to a conventional MSM-PD. Over the years, there have been many photonic investigations and researches performed to improve the performance of MSM-PDs by incorporating different nano-grating structures on top of the subwavelength aperture. Nano-gratings or nano-corrugations are metallic materials which have negative real permittivity. Metals like gold $(\mathrm{Au})$ or silver $(\mathrm{Ag})$ are usually used for constructing the nano-gratings [26]. For extraordinary optical transmission (EOT), excitation of surface plasmon polaritons (SPPs) is important. The SPPs confine the electromagnetic field in nanoscale to the central slit. These nano-gratings collect the incident light, falling on top of the device, and transmit the light efficiently to the central slit. The interaction between the photons with the confined electromagnetic field, inside the subwavelength aperture, causes light to reach a very high order of magnitude that opposes the standard aperture theory [8]. Hence, the device performance improves significantly since the transmission and absorption of the light to the active region of the device has enhanced to a great extent [9]. We have investigated the performance of MSM-PDs, which are assisted by different nano-corrugations, by varying the physical parameters of the plasmonic corrugated structures. We have used the Opti-FDTD simulation tool, based on the FDTD method, to investigate the effects of specific nano-structure on the MSMPDs and evaluate the improvement in the device performance in terms of light absorption enhancement.

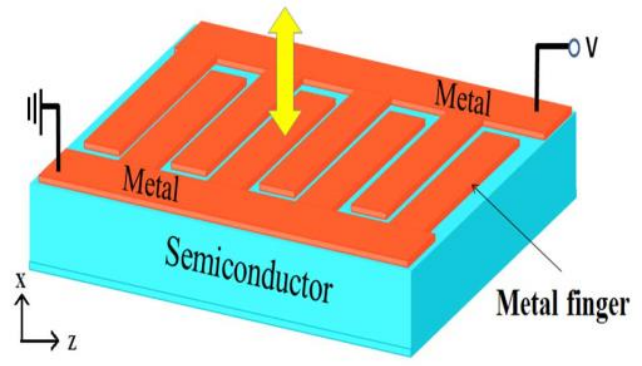

Fig. 1. Structural diagram of a conventional MSM-PD [13].

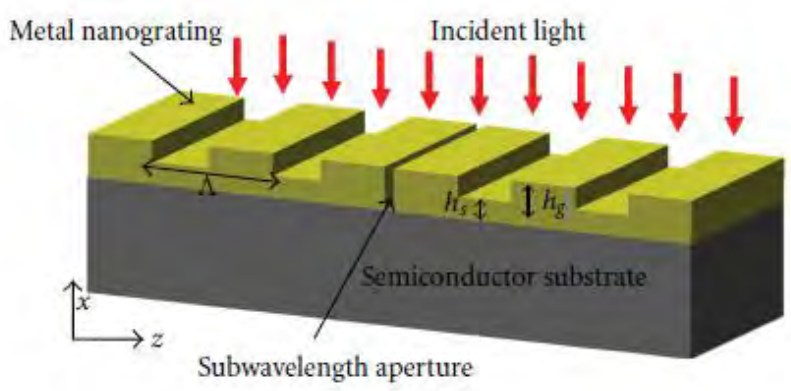

Fig. 2. Structure of a MSM-PD with rectangular shaped nano-gratings [21].

\section{DeVElopment of PlasmoniC-BASED MSM-PDS}

A conventional MSM-PD-structure is designed with two Schottky contacts on top of a semiconductor substrate. These contacts are called metal fingers, as shown in Fig.1. There are interdigitated metal electrodes (metal fingers) on top of the contacts, where plasmonic nano-gratings are placed. Gallium Arsenide (GaAs) is used as the semiconductor substrate in the device. It is used in most forms of optoelectronic applications. It has several benefits like having direct band gaps, higher electron mobility, and high-efficient light emissions for both electrical and optical applications [14]. The semiconductor substrate, used inside the device, should be consistent with the operation of the device in the selected wavelength range. GaAs is an ideal candidate for such application since it has a bandgap energy of
$1.42 \mathrm{eV}$, which corresponds to a De Broglie wavelength of 870$\mathrm{nm}$. Any incident light having greater wavelength than this will not be absorbed by the device. Hence, GaAs has the highest sensitivity for the desired wavelength range, i.e. 800-850-nm, of the developed devices since it has excellent quantum efficiency in this range. If the wavelength of the incident light falls below $800-\mathrm{nm}$, the quantum efficiency for the photodetectors decreases rapidly due to the recombination losses at the surface of the semiconductor substrate

As mentioned earlier, the application of plasmonic materials, on top of the device surface, leads to improve light transmission to the device. The material, gold ( $\mathrm{Au}$ ), is used in the MSM-PDs as a thin film inside the central slit, which contributes to significant SPP excitations [23, 24]. The importance of SPP excitation for EOT is second to none and studies related to it are still under investigation $[1,6]$. The nano-structured MSM-PD design consists of three separate parts; the top layer which contains the plasmonic nano-gratings, the middle layer which contains the subwavelength aperture, and the bottom layer which has undoped semiconductor substrate, as shown in Fig. 2. The surface of the metal contains nano-gratings that assist to concentrate the incident light in case of resonance. This concentrated light is transmitted to the surface of the semiconductor substrate through the subwavelength aperture. Thus, improves the light transmission and absorption in the device [8]. The device is subjected to electromagnetic wave and it travels, in the form of SPPs, over the surface of the metal. However, the SPPs cannot be excited by photons only and a coupling technique is needed [4]. Hence, nano-gratings are introduced which improves light absorption and enhancement inside the device [2]. The dimensions of these nano-gratings should be smaller than the wavelength of the incident light in order to contribute to the absorption enhancement. Thus, considering this scenario, the electromagnetic field is strongly confined by the metal surface and the coupling process of SPPs is improved significantly [7]. In a metal-dielectric interface, the SPP wave vector matching condition for a metal nano-grating is defined by the following equation,

$$
k_{s p p}=\frac{\omega}{c} \sin (\theta) \pm \frac{2 \pi l}{\Lambda}=\frac{\omega}{c} \sqrt{\frac{\varepsilon_{m}^{\prime} \varepsilon_{d}}{\varepsilon_{m}^{\prime}+\varepsilon_{d}}}
$$

where ' $\omega$ ' represents the angular frequency of the incident light wave, ' $\theta$ ' represents the angle of light incident to the device, ' $c$ ' represents the speed of light in vacuum, ' $l$ ' is an integer number i.e., $l=1,2,3, \ldots, \mathrm{N}$ and represents the metal nano-grating period.

The purpose of this research is to model and analyze the performance of MSM-PDs employed with different nanograting shapes. The physical parameters of the nano-gratings are varied to achieve the maximum light absorption by the device, i.e. the device will have maximum sensitivity in the selected wavelength of light (830-nm). Fig. 3 illustrates the diagram of a designed model of a nano-grating assisted MSM-PD in the OptiFDTD simulation tool. It shows the parameters that were varied to optimize device performance. The nano-grating shapes used for this research are given in Fig. 4. It shows the schematic diagrams of nano-structured MSM-PDs consisting of (I) trapezoidal, (II) triangular, (III) hemispherical, and (IV) rectangular shaped nano-gratings. These improved devices can contribute to faster communication systems with their superior light sensitivity. They are also useful for sensor networks. 


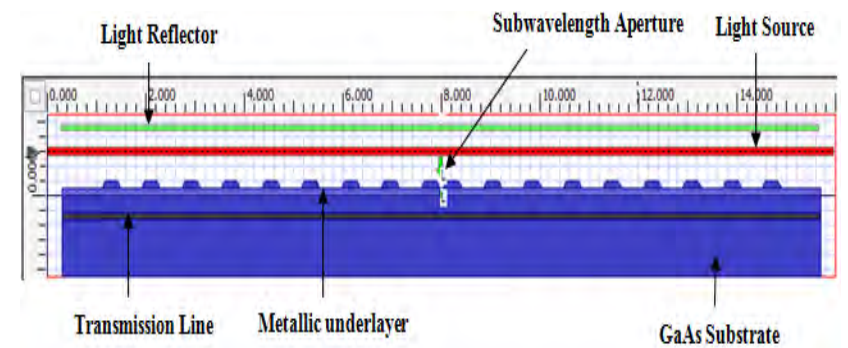

Fig. 3. Detailed diagram of the simulation model.

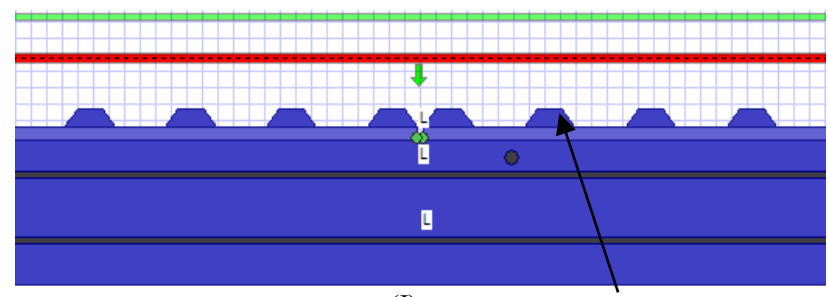

(I) Trapezoidal Shaped Nano-gratings

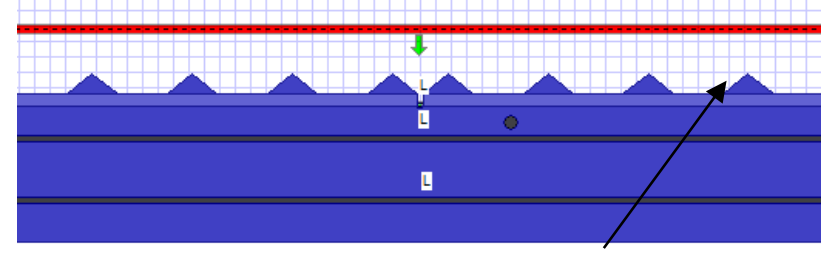

(II) Triangular Shaped Nano-gratings

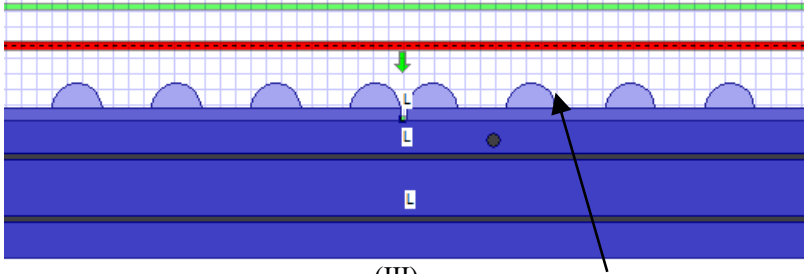

(III) Hemispherical Shaped Nano-gratings

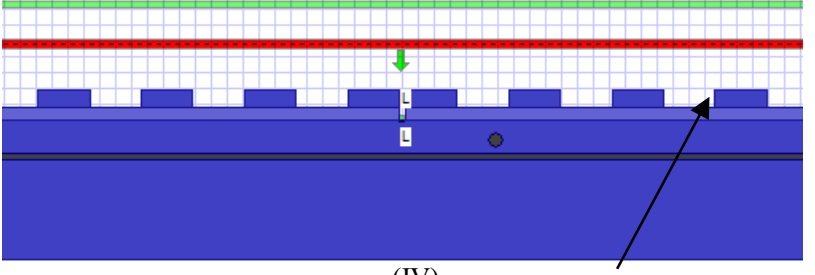

(IV) Rectangular Shaped Nano-gratings

Fig. 4. Schematic diagram of (I) Trapezoidal, (II) Triangular, (III) Hemispherical, and (IV) Rectangular shaped nano-gratings.

\section{Simulation SETUP FOR MSM-PD STRUCTURES}

This section discusses the simulation set-up for the MSMPDs employing different nano-grating structures. For the simulation of plasmonic nano-structured MSM-PDs, we adopted the Opti-FDTD simulation tool that is based on the FDTD method. This simulation tool is useful for analyzing the power spectrum of the developed MSM-PDs. Fig. 5 represents the flowchart of the simulation process in the Opti-FDTD tool. The flowchart shows the design model and analysis of MSM-PDs by using the Opti-FDTD software. The procedure is used for each MSM-PD structure with different nano-gratings intended for this study. The wavelength of the incident light, which is impinging on the nano-grating structures, is $830-\mathrm{nm}$ and is a Gaussian modulated continuous wave. It is important to employ an appropriate model to specify the dielectric properties of the plasmonic materials because below plasma or resonance frequency, plasmonic materials like gold $(\mathrm{Au})$ and silver $(\mathrm{Ag})$ have negative real permittivity. This is because at the plasma frequency, $\omega_{p}$, the dielectric function of such bulk materials tend to change their sign and the real part of it becomes zero [26]. When this occurs, the following equation can be applied to calculate the plasma frequency of the specific material,

$$
\omega_{p}^{2}=\frac{n \cdot q_{e}^{2}}{\varepsilon_{o} \cdot m_{e}}
$$

where ' $\omega_{p}$ ' is the plasma or resonance frequency, ' $n$ ' is the no. of electrons per unit volume, ' $q_{e}$ ' is the charge of an electron, ' $\varepsilon_{o}$ ' is the permittivity in real space, and ' $m_{e}$ ' is the mass of an electron. As mentioned earlier, the plasmonic material used for modeling the devices is gold $(\mathrm{Au})$. Its dielectric and magnetic properties are taken from the Lorentz-Drude model [16]. The dielectric function of the material can be expressed by [12]:

$$
\varepsilon_{\mathrm{r}}(\omega)=\varepsilon_{\mathrm{r}}^{\mathrm{f}}(\omega)+\varepsilon_{r}^{b}(\omega)
$$

where, ' $\varepsilon_{r}(\omega)$ ' represents the complex dielectric function of metals, ' $\varepsilon_{r}^{f}(\omega)$ ' represents the effects of free electrons and ' $\varepsilon_{r}^{b}(\omega)$ ' represents the effects of bound electrons in the metal. Hence, this model takes into account both intraband, $\varepsilon_{r}^{f}(\omega)$ and interband, $\varepsilon_{r}^{b}(\omega)$ electronic transitions for simulations [27]. The metal corrugated grooves, i.e. the nano-gratings, energize the SPPs from both positive and negative sides. The power of the SPPs wave decreases with distance. This restrains the noncentral grooves to energize the SPPs and spread towards the subwavelength apertures. Thus, the coupling of incident light with SPP energized by non-central notches of the nano-gratings is restricted by this condition. So the most dynamic piece of the nano-gratings is the incorporated ones (central ones). They act like wave collectors causing resonance absorption for the MSMPDs. This happens when the energized SPPs couple with the electromagnetic field inside the subwavelength aperture. This coupled wave is transmitted to the active part of the device, i.e. in the semiconductor substrate, through the central slit or subwavelength aperture [25]. Electron-hole pairs are produced in GaAs semiconductor substrate when the coupled wave is transmitted to it. This energizes SPPs close to the metalsemiconductor intersection which enhances the light ingestion of the gadget [13]. This happens on the grounds that light transmission expands, which increment the absorption of the GaAs substrate. The LAEF is defined as the standardized power transmittance of the MSM-PD with nano-gratings to the MSMPD without nano-gratings. This upgrade is very useful with the geometry of metal gratings [15]. The diverse geometry of the nano-gratings influences the performance of the MSM-PDs regarding light absorption. Light absorption likewise relies upon the width of the subwavelength aperture. The slit width should be smaller than the wavelength of the incident light to accomplish symmetric and fundamental surface plasmon modes, which will spread toward the slit. Subsequently, light transmission, towards the semiconductor substrate, relies upon the width of the subwavelength cut. Henceforth it is imperative to improve the slit width to increase the LAEF since smaller slit width helps in accepting and control of the incident light. For the demonstrating of MSM-PDs employed with nano-corrugations, slit width of 50 500-nm was utilized in this study. A slit width longer or shorter then this chosen range won't contribute a lot to the confinement of incident light i.e., to the light absorption. Also, the fabrication procedure will be troublesome and probably won't be productive. Also, narrower slit width makes the absorption efficiency to increase, which was observed in the LAEF spectra of the devices provided in the result section. This prompts the higher light transmission inside the active region of the device [10]. 


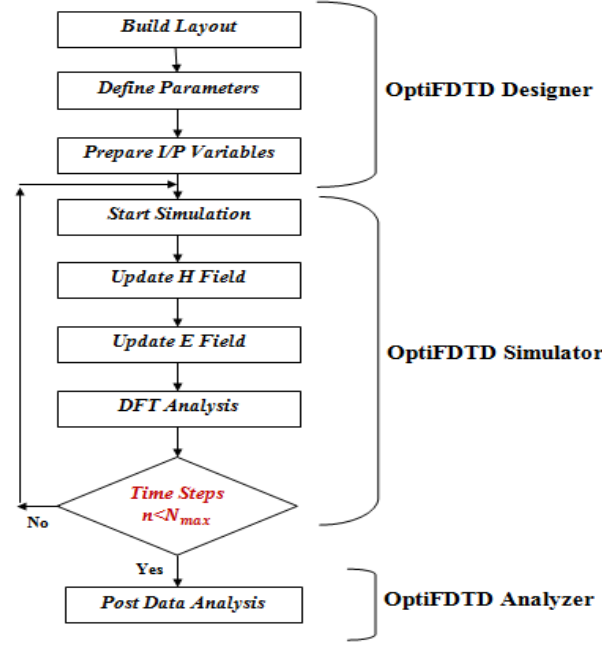

Fig. 5. Flowchart of the simulation process for modeling and analyzing the devices [20].

\section{SimULATION RESUlTS AND DISCUSSION}

The simulation results are presented in this section in brief. Here, several nano-structured MSM-PDs simulation results are given and analyzed in details. The actual size of the device affects the confinement of incident light in the active region, i.e. the coupling process, as well as the light absorption, is affected by the size of the photodetector. Hence, the performance of the MSM-PD is significantly improved by incorporating plasmonic nano-gratings on top of the device surface for improved light transmission in the active region of the device. The application of the nano-gratings will optimize the device performance significantly when their geometry is optimized for enhanced light absorption. The results obtained from the simulation of plasmonic-based MSM-PDs were compared with conventional MSM-PDs to calculate the enhancement of light absorption. Table 1 is listed with the nano-grating parameters used for the optimization of the device.

TABLE 1- PARAMETERS USED FOR OPTIMIZATION OF NANOGRATINGS.

\begin{tabular}{ll}
\hline Parameters & Values \\
\hline
\end{tabular}

Grating period

$810-\mathrm{nm}$

Nano-gratings on each side

Nano-grating height

100 200-nm

Slit width

$50 \sim 500-\mathrm{nm}$

Subwavelength aperture height

$60-\mathrm{nm}$

Duty cycle

$50 \%$

\section{A. Trapezoidal-shaped Nano-structured MSM-PDs}

In this sub-section, the light absorption enhancement in an MSM-PD with optimized trapezoidal nano-gratings is discussed. The aspect ratio used for modeling the trapezoidal nano-gratings is 0.5 and the rest of the parameters are kept constant as mentioned in Table 1. Fig. 6 represents the LAEF for the trapezoidal nano-grating assisted MSM-PD. The figure gives evidence to the concept that, narrower slit width gives better light absorption compared to wider slit width since, at one instance of the simulation process, the trapezoidal nano-grating assisted MSM-PD achieved about 34-times LAEF for a slit width of 50-nm while the minimum LAEF was obtained for a slit width of 500-nm, which is about 2.4-times when compared with conventional MSM-PDs. Hence, the device was able to gain maximum light absorption for the 50 -nm slit. Fig. 7 also represents the LAEF for trapezoidal nano-structured MSM-PDs. But here the slit width is kept constant at $50-\mathrm{nm}$ and the grating height is varied. Other parameters are kept constant. The plot provides proof to the fact that the height of the nano-grating is also an important aspect of the device performance. But, there is no rule of thumb to obtain the most optimized height for the device. It affects the LAEF of a device with corresponding changes in other grating parameters along with it. The waveform shows that at a nano-grating height of $100-\mathrm{nm}$, the device was able to achieve the maximum LAEF, which is about 34-times. The minimum LAEF was obtained by a device with a $160-\mathrm{nm}$ nano-grating height, which is about 6-times. These results show unusual behavior for the MSM-PDs with trapezoidal nanogratings on top of them since theoretically, higher nano-grating height should be able to attain a large amount of light. But in reality, that was not the case. One reason for such results might be due to the fact that taller trapezoidal shapes have shorter elliptical walls, causing more light to refract than to guide them toward the subwavelength apertures.

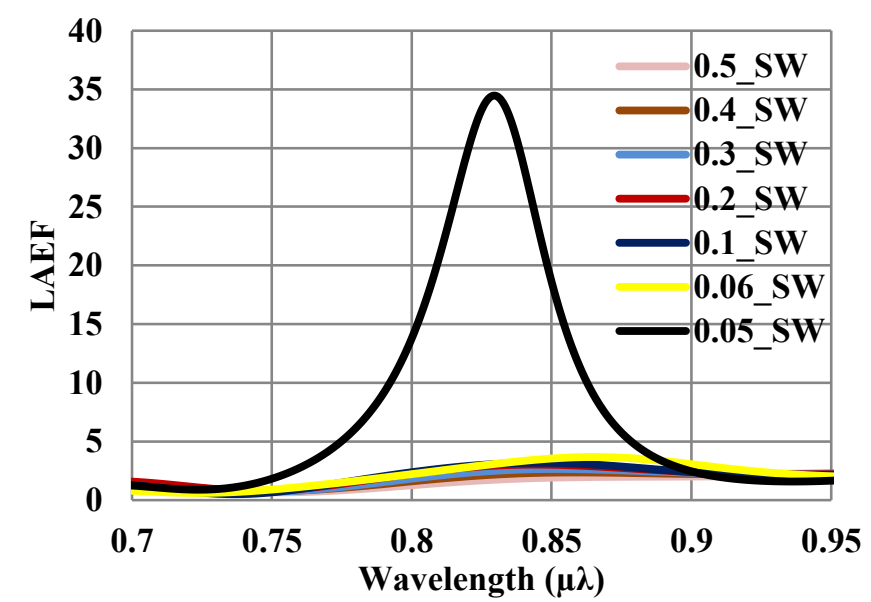

Fig. 6. LAEF for trapezoidal nano-structured MSM-PD with constant height.

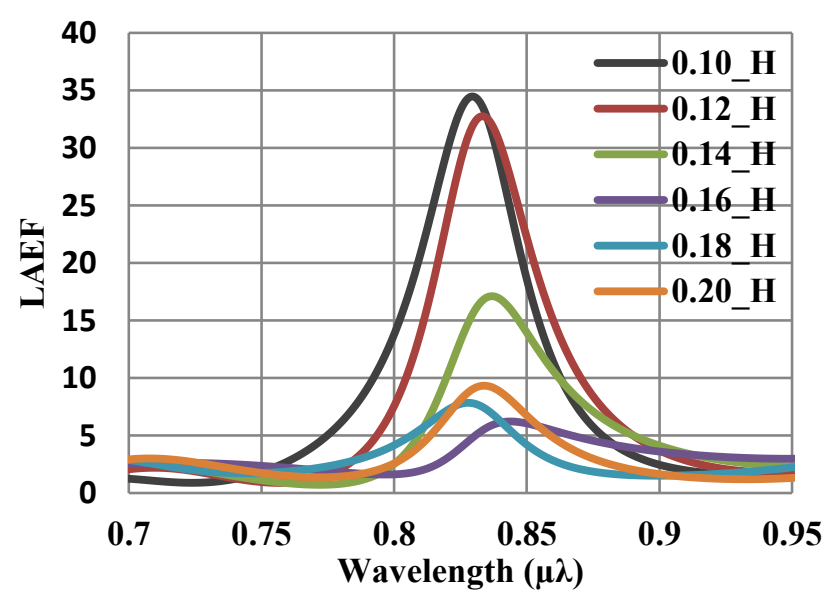

Fig. 7. LAEF for trapezoidal nano-structured MSM-PD with constant slit width.

\section{B. Triangular-shaped Nano-structured MSM-PDs}

In this sub-section, the light absorption enhancement in an MSM-PD with optimized triangular nano-gratings is discussed. The aspect ratio is zero while other parameters are kept constant. Fig. 8 shows the LAEF for the triangular nano-grating assisted MSM-PD. The figure again gives evidence to the fact that narrower slit width gives better light absorption compared to wider slit width since, the maximum LAEF of about 31-times, 
was achieved for a slit width of 50-nm while the minimum LAEF of about 2.7-times, was found for 500-nm slit width. Fig. 9 represents the LAEF for triangular nano-structured MSM-PDs. Here the slit width is kept constant at $50-\mathrm{nm}$ and the grating height is varied while keeping other parameters constant. The plot shows a maximum LAEF of about 37-times was achieved by an MSM-PD with triangular nano-gratings of height $180-\mathrm{nm}$, while the minimum LAEF of about 28-times was achieved for a grating height of $120-\mathrm{nm}$. The most successful result was achieved by the triangular nano-grating assisted MSM-PD with a grating height of $180-\mathrm{nm}$ and slit width of 50-nm since it was able to achieve the maximum result out of all the nano-structured MSM-PDs. The reason behind such an excellent result is because of the fact that taller triangular shape will confine more light inside the subwavelength aperture because of its steeper edges. Hence, light transmission and absorption are improved for MSM-PDs employing such nano-structures.

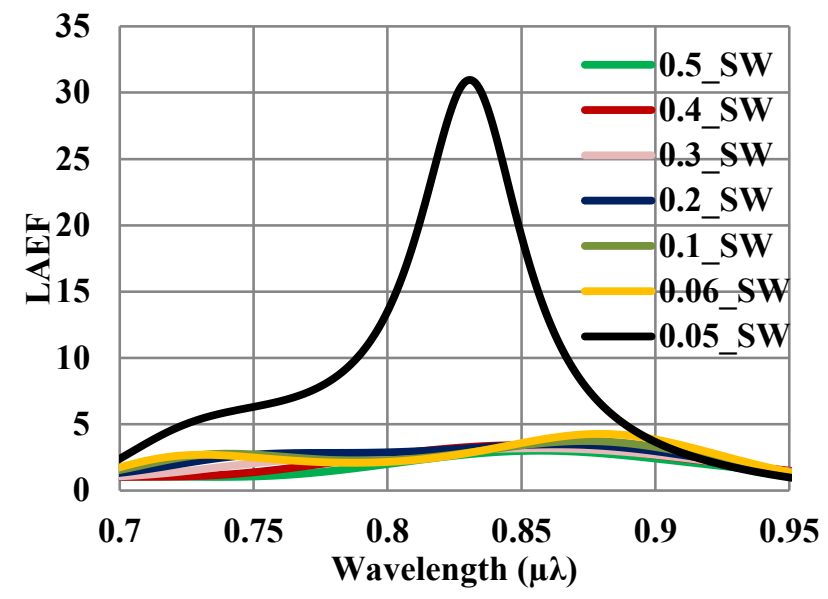

Fig. 8: LAEF for triangular nano-structured MSM-PD with constant height.

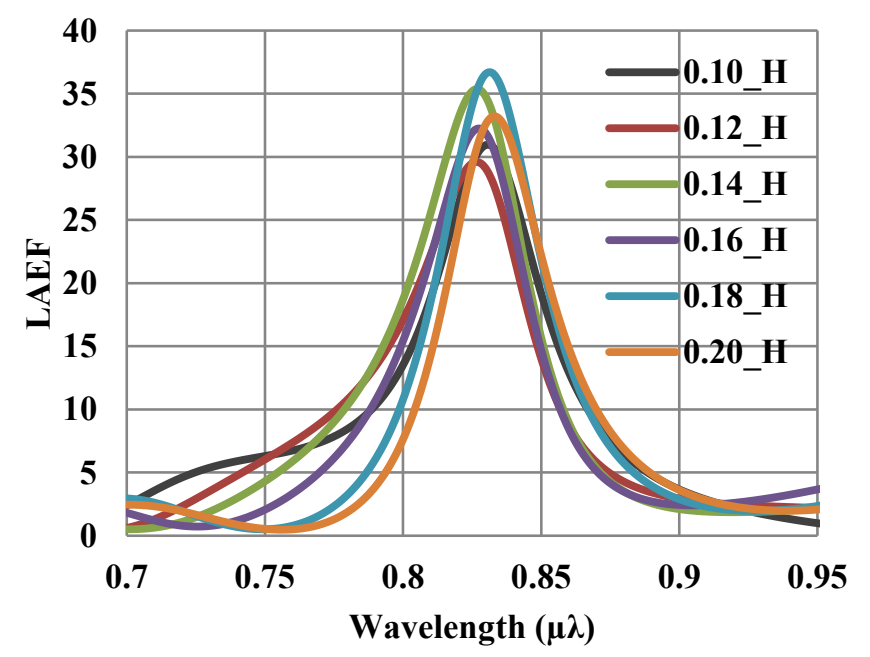

Fig. 9. LAEF for triangular nano-structured MSM-PD with constant slit width.

\section{Hemispherical-shaped Nano-structured MSM-PDs}

In this sub-section, the light absorption enhancement in an MSM-PD with optimized hemispherical nano-gratings is discussed. This is a newly developed nano-grating structure used in this research. While fabricating devices with such small dimensions, i.e. in the nanometer range, it is important to maintain the nano-grating geometry accurately. But usually, the geometry of the nano-gratings are not fully perfect and various scenarios of change of geometrical shape might take place. Hemispherical nano-gratings aid the fabrication process since no complex geometry is needed to develop such shapes. Fig. 10 shows the LAEF for the hemispherical nano-grating assisted
MSM-PD. The grating height is one-quarter of the grating period which is also the radius of the hemisphere. Hence for a nanograting period of $810-\mathrm{nm}$, the height of the hemispherical nanograting is 202.5-nm which is kept constant for this plot. The subwavelength aperture height used in this modeling is $100 \mathrm{~nm}$. The figure shows that narrower slit gives better light absorption since the maximum LAEF was achieved by the device employing it, which is about 19.5-times for a slit width of $50-\mathrm{nm}$ while the minimum LAEF of about 2.4-times was achieved for a slit width of 500-nm. Currently, it is not possible to vary the height of this type of nano-grating shape without changing the grating period. Hence, only the slit width variation plot is given.

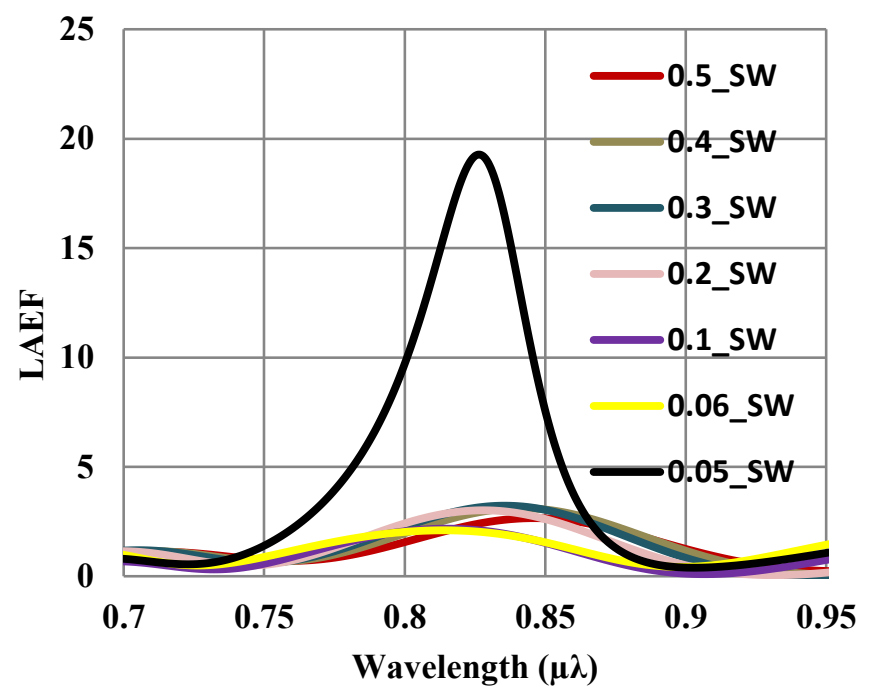

Fig. 10: LAEF for hemispherical nano-structured MSM-PD with constant height.

\section{Rectangular-shaped Nano-structured MSM-PDs}

In this sub-section, the light absorption enhancement in an MSM-PD with rectangular nano-gratings is discussed. The parameters of the rectangular nano-gratings are optimized for improved LAEF in the device. Fig. 11 represents the LAEF for the rectangular nano-grating assisted MSM-PD. The grating height is kept constant to 100 -nm while varying the slit width. The device was able to achieve a LAEF of about 16.2-times for a slit width of 50-nm while it achieved the minimum LAEF of about 2.2-times for a 500-nm slit width. Fig. 12 represents the LAEF for rectangular nano-structured MSM-PDs. Here the slit width is kept constant at 50-nm and the grating height is varied while keeping other parameters constant. Here, a nano-grating height of $100-\mathrm{nm}$ gave the maximum LAEF, i.e. about 16.2times for the device while the nano-grating height of $160-\mathrm{nm}$ gave the minimum LAEF, i.e. about 1.5-times. The results obtained are similar to results observed for the trapezoidal nanostructured MSM-PD. This is because the structures are similar in geometry apart from the different aspect ratio. This is why it achieved such lower outcomes.

\section{E. Comparison of LAEF for Different Nano-Structured MSM-PDs}

As we have observed in previous sub-sections, different nano-structured MSM-PDs were able to achieve the maximum or highest LAEF for a slit width of 50-nm. Fig. 13 shows the LAEF spectra of each nano-structured MSM-PD with 50-nm slit width. From this plot, it is observed that the trapezoidal nanograting achieved the highest LAEF of 34-times compared to a conventional MSM-PD. The reason behind such a result is due to the fact that nonlinear nano-grating shapes effectively improve the collection of light and aid in guiding it to the central aperture [7]. However, one thing is certain here, the height of the nano-gratings was kept constant to $100-\mathrm{nm}$. But better LAEF was achieved by the triangular nano-grating assisted MSM-PD 
for a height of $180-\mathrm{nm}$ while the trapezoidal nano-structured MSM-PD failed to achieve better results for such nano-grating height.

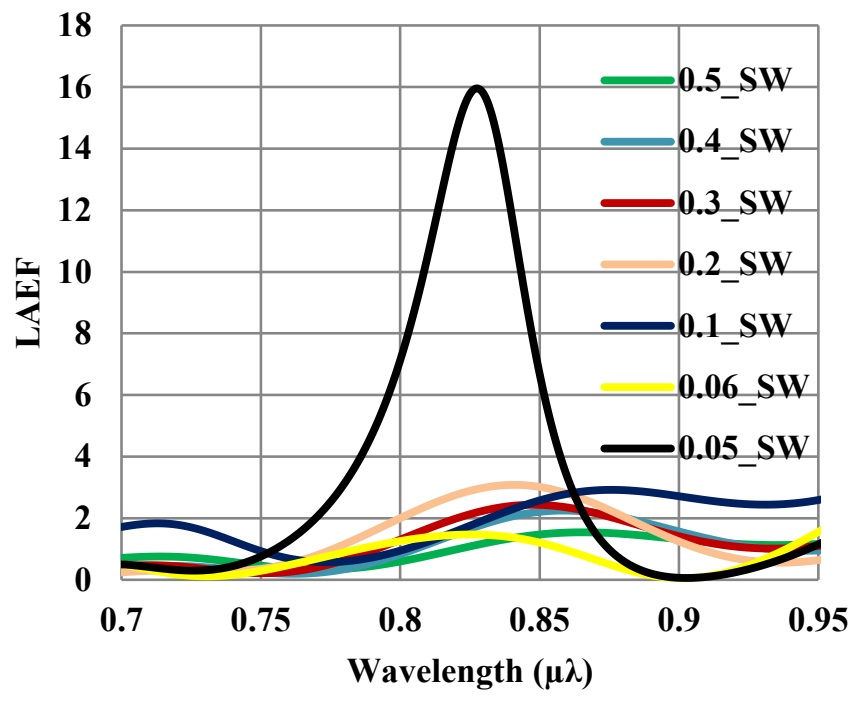

Fig. 11. LAEF for rectangular nano-structured MSM-PD with constant height

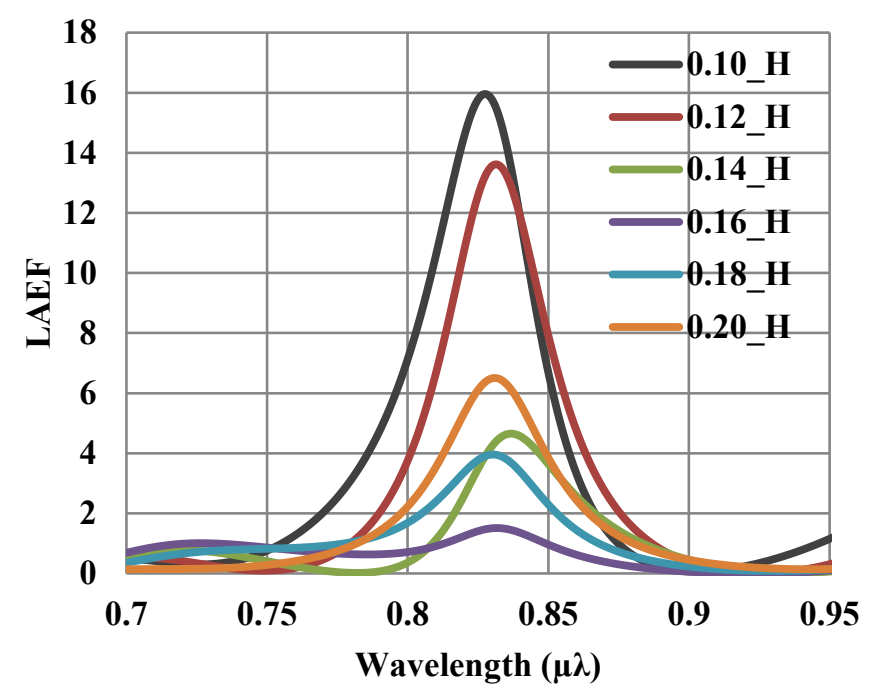

Fig. 12. LAEF for rectangular nano-structured MSM-PD with constant slit width.

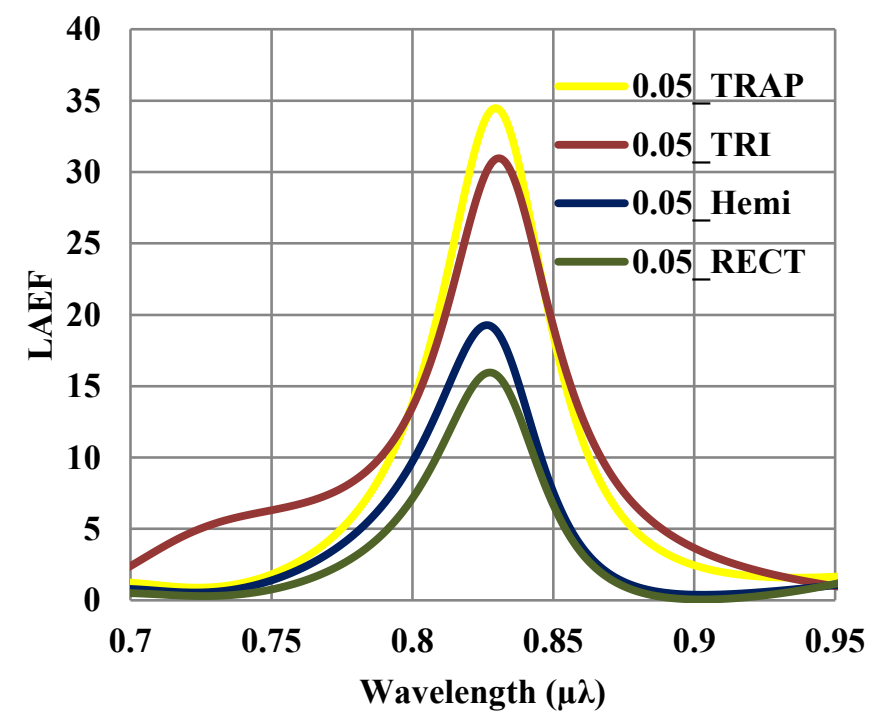

Fig. 13. LAEF for every nano-structure assisted MSM-PDs with constant slit width.

\section{F. Fabrication Difficulties for the Proposed Nano-structured MSM-PDs}

This section discusses the fabrication process and its difficulties related to the developed nano-corrugated MSM-PDs. The results obtained from the simulated devices are based on one condition; the nano-grating shapes will remain unchanged throughout the fabrication process. This helped to investigate the performance of these nano-scaled devices in a controlled environment, i.e., at normal temperature, seamless interaction between the incident light and the device, perfect phase shift of slits, and proper deposition of the plasmonic material (i.e., gold) during the lithography process. But, in a practical scenario, the shape of the nano-gratings depends on the fabrication technology [28]. In most cases, the nano-grating structures changes during the photo-lithography process. The edges or ridges of the nano-gratings gets truncated easily during the etching operation. To avoid this complexity, a more sophisticated lithography procedure is used, which is known as the focused ion beam (FIB) lithography. It has superior performance compared to conventional lithography on cutting sharp-edge profiles [9. 21]. Hence, it is better to execute the fabrication operation for the developed devices using the FIB lithography process. The success of etching the perfect nanograting structure also depends on the complexity of the geometry for that structure. For example, it will be easier to etch the hemispherical structure compared to other mentioned nanograting structures. This is because, it requires a simpler procedure to etch hemispherical shapes compared to triangular, rectangular, and trapezoidal shapes or structures. In addition, the sharp edges of the above-mentioned structures might not remain sharp during the etching process. This reduces the percentage yield of the procedure. The performance comparison of the developed devices was made with respect to the changes in their geometrical parameters, i.e., slit width, nano-grating height. The impact of each parameter on the LAEF of the devices was investigated while keeping the other parameters constant. For example, the investigation of the impact of changes in the nano-grating height was executed while keeping the slit width constant. This limits the performance of the developed devices. Also, with increasing the nano-grating heights, the percentage yield of the FIB lithography process decreases further. Hence, better optimization of each nanograting parameters for further improvement in the device performance is needed before going to the fabrication process. This is an important area of research for future research and development studies.

\section{CONCLUSION}

We have designed, modeled and analyzed the influence of different types of nano-structured MSM-PDs in this research to obtain the maximum light transmission and absorption into the active region of the MSM-PDs. The effect of the employed nanogratings, on top of the device, for higher light transmission is observed in the simulation results. Evidence regarding the interaction of light with the SPPs to achieve a higher order of magnitude was observed since the modeled devices showed excellent performance under several simulation conditions. The geometry of the applied nano-gratings was varied to optimize the performance of the MSM-PDs. Parameters such as slit width, grating height, subwavelength aperture height, aspect ratio, and duty cycle were optimized to get the maximum LAEF. The effect of slit width and nano-grating height variation on the LAEF of the MSM-PD was studied in details. The simulation results gave evidence to the fact that narrower slits aid in higher confinement of light in the active region of the device. Hence, light transmission and absorption improved notably. The nano- 
grating height was varied for each plasmonic structure to observe their effect on the light absorption. But, there was no general pattern observed by changing the height of the nano-grating shapes, i.e., different nano-structures affected the MSM-PDs differently to the change in their height. However, the highest LAEF was achieved by the triangular nano-grating assisted MSM-PD for a height of $180-\mathrm{nm}$ with $50-\mathrm{nm}$ slit width. It has achieved about 37-times higher LAEF compared to conventional MSM-PDs. Furthermore, a significant light absorption enhancement was found for trapezoidal and hemispherical shaped nano-grating assisted MSM-PDs. The simulation results demonstrated that the responsivity of these plasmonic-based MSM-PDs has improved considerably since the nano-grating geometry was optimized for improved performance of the MSM-PDs. These improved devices are suitable for applications such as high-speed communication systems as well as faster sensor network systems.

\section{ACKNOWLEDGMENT}

This research was supported by the Department of Electrical and Electronic Engineering, Faculty of Engineering, American International University-Bangladesh (AIUB), Dhaka-1229, Bangladesh.

\section{REFERENCES}

[1] J. B. D. Soole and H. Schumacher, "InGaAs metal-semiconductor-metal photodetectors for long wavelength optical communication," IEEE J.Quantum Electron., vol. 27, no. 3, pp. 737-752, Mar. 1991.

[2] D. R. Scherer, P. M. Mayer, J. M. Hensley, M. G. Allen, P. Mukherjee, and M. Schattenburg, "Plasmonic absorption enhancement in organic photovoltaic cells with periodic metallic nanostructures," in: Proc. of the CLEO 2011 - Laser Applications to Photonic Applications, OSA Technical Digest (CD) Optical Society of America, 2011.

[3] J. Hetterich, G. Bastin, N. A. Gippius, S. G. Tikhodeev, G. von Plessen, and U. Lemmer, "Optimized design of plasmonic MSM photodetector," IEEE J.Quantum Electron., vol. 43, No. 10, 2007.

[4] [4] F. F. Masouleh, N. Das, and H. R. Mashayekhi, "Assessment of amplifying effects of ridges spacing and height on nano-structured MSM photo detectors," Opt. and Quant. Electron.,vol. 47(2), pp.193-201, 2015.

[5] M. Y. Liu and S. Y. Chou, "Nanoscale metal-semiconductor-metal photodetectors with subpicosecond response time fabricated using electron beam lithography," J. Vac. Sci. Technol. B 10, 2932-2935, 1992.

[6] Y. Ding, J. Yoon, M. H. Javed, S. H. Song, and R. Magnusson, "Mapping Surface-Plasmon Polaritons and Cavity Modes in Extraordinary Optical Transmission," IEEE Photonics Journal, vol. 3, No. 3, 2011.

[7] F. F. Masouleh, N. Das, and H. R. Mashayekhi, "Comparison of different plasmonic nanograting profiles for quality light absorption in nanostructured metal-semiconductor-metal photodetectors," Opt. Eng. 52(12) 127101, 2013.

[8] N. Das, F. F.Masouleh, and H. R. Mashayekhi, "Light absorption and reflection in nanostructured GaAs metal-semiconductor-metal photodetectors," IEEE Trans. on Nanotech, vol. 13, No. 5, 2014.

[9] F. F. Masouleh and N. Das, "Application of Metal-Semiconductor-Metal Photodetector in High-Speed Optical Communication Systems," Advances in Optical Communication, 2014.

[10] F. F. Masouleh, N. Das, and H. R. Mashayekhi, "Optimization of light transmission efficiency for nano-grating assisted MSM-PDs by varying physical parameters," Phot. Nano.Fund. Appl., vol. 12(1), pp.45-53, 2014.

[11] F.F. Masouleh, N. Das, S.M. Rozati, “Optimal subwavelength design for efficient light trapping in central slit of plasmonics-basedmetalsemiconductor-metal photodetector," Opt. Quant. Electron. 47, 1477$1485,2015$.

[12] A. D. Rakic, A. B. Djurišic, J. M. Elazar, and M. L. Majewski, "Optical Properties of Metallic Films for Vertical-Cavity Optoelectronic Devices," Applied Optics; 37(22), 5271-5283, 1998.

[13] N. Chandan, N. Das and F. F. Masouleh, "Analysis of plasmonics-based nano-structured MSM-PDs for enhanced light absorption," proc. of the $11^{\text {th }}$ International Conference on Sensing Technology 2017 (ICST 2017), pp.1-5,Macquarie Univ., Sydney, NSW, Australia, 2017.

[14] J. Yoon, S. Jo, I. Chun, I. Jung, H. Kim, M. Meitl, E. Menard, X. Li, J. Coleman, U. Paik and J. Rogers, "GaAs photovoltaics and optoelectronics using releasable multilayer epitaxial assemblies," Nature, vol. 465, no. 7296, pp. 329-333, 2010.

[15] F.F. Masouleh, S.M. Rozati, N. Das, "Performance improvement of plasmonic-based thin film assisted MSM-PDs," Optik, Volume 157,Pages 733-742,ISSN 0030-4026, 2018.

[16] R. Umeda, C. Totsuji, K. Tsuruta, and H. Totsuji, "Dispersion Models and Electromagnetic FDTD Analyses of Nanostructured Metamaterials using Parallel Computer," Memoirs of the Faculty of Engineering, Okayama University; 43, p. 8, 2009.

[17] C. L. Tan, V. V. Lysak, N. Das, A. Karar, K. Alameh, and Y. T. Lee, "Absorption enhancement of MSM photodetector structure with a plasmonic double grating structure," in proc. of the 10th Int'l Conf. on Nanotech. 'IEEENANO 2010', Seoul, S. Korea, 2010.

[18] I. M. Mehdi Hasan, "FDTD Analysis of Plasmonic and Nanojet Enhanced Photodetectors for Improved Performance," Masters Dissertation, The University of New Mexico, Albuquerque, NM, USA, 2012.

[19] Z. Fan, Y. Su, H. Zhang, X. Han, and F. Ren, "Analysis of aluminum nanogratings assisted light reflection reduction in GaAs metalsemiconductor metal photodetectors," in Proc. of the SPIE 9563, Sept. 2015.

[20] Opti-FDTD, 12th ed. Optiwave, Ottawa, Ontario, Canada, 2014.

[21] N. Das, A. Karar, C. L. Tan, K.Alameh, Y. T. Lee, "Impact of Nanograting Phase-Shift on Light Absorption Enhancement in Plasmonics-Based Metal-Semiconductor-Metal Photodetectors," Advances in Optical Technologies, 2011.

[22] G. P. Wiederrecht, "Handbook of nanoscale optics and electronics," Elsevier, Amsterdam, First edition 2010. ISBN: 978-0-12-375178-2.

[23] B. Sturman, E. Podivilov, and M. Gorkunov, "Theory of extraordinary light transmission through arrays of subwavelength slits,"Phys. Rev. B, 77, 075106, 2008.

[24] S. Kim, Y. Lim, H. Kim, J. Park, and B. Lee, "Optical beam focusing by a single subwavelength metal slit surrounded by chirped dielectric surface gratings," Appl. Phys. Lett. 92 (013103), 1-3, 2018.

[25] J. A. Porto, F. J. García-Vidal, and J. B. Pendry, “Transmission resonances on metallic gratings with very narrow slits," Physical Review Letters, 83, 2845-2848, 1999.

[26] A. Derkachova, K. Kolwas, and I. Demchenko, "Dielectric Function for Gold in Plasmonics Applications: Size Dependence of Plasmon Resonance Frequencies and Damping Rates for Nanospheres,' Plasmonics, 11: 941, 2016.

[27] A. D. Rakić, "Algorithm for the determination of intrinsic optical constants of metal films: application to aluminum," Appl. Opt. 34(22), 4755-4767, 1995.

[28] W. Kuo, "Challenges related to reliability in Nano electronics", IEEE Trans. Reliability, vol. 55, no. 4, pp. 569-570, 2006.

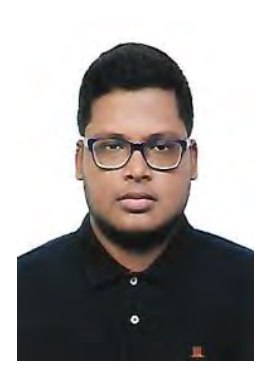

Alif Islam received B.Sc. degree in Electrical and Electronic Engineering from American International University-Bangladesh (AIUB), Dhaka, Bangladesh in 2018. He is currently pursuing his M.Sc. degree in Electrical and Electronic Engineering from AIUB, Dhaka, Bangladesh. His research interest includes robotics, power electronics, microelectronics, quantum mechanics, photonics, renewable technology, and power system.

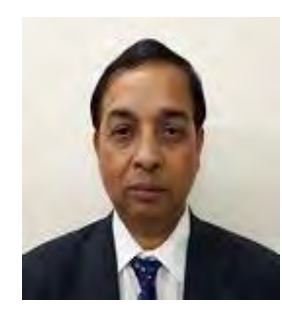

Narottam Das received B.Sc. degree in Electrical and Electronic Engineering from Chittagong University of Engineering and Technology, Chittagong, Bangladesh in 1992, M.Sc. degree in Electrical and Electronic Engineering from Bangladesh University of Engineering and Technology (BUET), Dhaka, Bangladesh in 1996, and PhD (Dr. Eng.) degree in Electrical Engineering from Yamagata University, Yamagata, Japan in 2000. His PhD research thesis was sponsored by the Japanese Government (Monbusho) Scholarship. Currently, Dr Das is working at Central Queensland University as a Senior Lecturer in Electrical Engineering, Melbourne Campus, Victoria, Australia. Prior, he worked as academic staff in Electrical and Electronic Engineering at University of Southern Queensland, Curtin University, Edith Cowan University, Monash University, Australia and American International University-Bangladesh (AIUB), Dhaka. Earlier, he worked at NEC Yamagata Ltd., Japan as a senior design Engineer and Bangladesh Export Import Company, Dhaka, Bangladesh. In 1993-1994, he worked as a Teaching Assistant/Research 
Assistant and Engineer at BUET, Dhaka, Bangladesh. Dr. Das is the author/co-author of 8 book chapters, over 150 peer-reviewed journal and international conference papers. He has presented his research works worldwide (more than 20 countries) as speaker and invited/ keynote speaker/ session chair. Dr. Das is a senior member of the IEEE PES and PHS, USA; Fellow of the Institution of Engineers, Australia; C.P. Eng., and Life Fellow of the Institution of Engineers, Bangladesh. His research interests include in Power Systems Communications (i.e., Smartgrids) using IEC 61850, Multi-junction Solar (PV) cells, modeling of high efficiency solar cells (renewable energy), and high-speed communication devices (SOAs and MSM-PDs). He is a regular reviewer of IEEE Transaction papers, IET papers, Elsevier, Energies, other similar Journal and conference papers. Dr. Das served as the program committee member of several IEEE conferences nationally and internationally as well as presented as an invited speaker. Dr. Das is the editor of the books Nanostructured Solar Cells, Advances in Optical Communication, Optical Communication, and Optical Communication Systems. He is also a Guest Editor of the Journal "Energies" Special Issue on "Nano-structured Solar Cells".

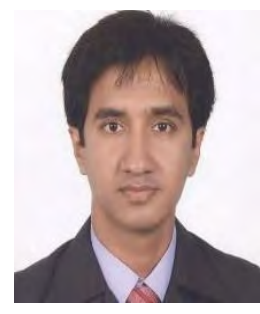

Mohammad M Uzzal received B.Sc. degree in Electrical and Electronic Engineering from BUET, Dhaka, Bangladesh in 2002 and M.S.E. degree in Electrical Engineering from Arizona State University (ASU), USA in 2006. He is currently working as a research assistant while pursuing a $\mathrm{Ph} . \mathrm{D}$. degree in micro-electronics with a research focus in mixed signal CMOS circuits for Biosensing application at University of New Mexico, NM, USA. From 2004 to 2012, he was with American International University - Bangladesh as a Full-time Faculty in Electrical and Electronic Engineering Department. From May to Dec. 2014 he was with Intel Corporation, Santa Clara, CA and worked on next generation high speed (10-16Gbps) $\mathrm{I} / \mathrm{O}$ design and simulation. His research interest includes analog/RF and mixed signal circuit design, low cost biosensors and nano-electronics. He holds a professional MBA degree from Institute of Business Administration, University of Dhaka, in 2008. 